\title{
SEASONAL TREND OF FEVER CASES SEEKING HEALTH CARE SERVICES AT A TERTIARY CARE CENTRE- AN AUDIT OF RETROSPECTIVE DATA FROM SUB-HIMALAYAN REGION
}

\author{
Piyush Sharma1, Mitasha Singh², Manoj Kumar Gandhi³, Sujeet Raina4, Sunil Kumar Raina5, Ashok Kumar Bhardwaj ${ }^{6}$ \\ 1Senior Research Fellow, Department of Community Medicine, Dr. Rajendra Prasad Government Medical College, Kangra, H. P. \\ ${ }^{2}$ Assistant Professor, Department of Community Medicine, ESIC Medical College and Hospital, Faridabad, Haryana. \\ 3Junior Resident, Department of Community Medicine, Dr. Rajendra Prasad Government Medical College, Kangra, H. P. \\ ${ }^{4}$ Assistant Professor, Department of Medicine, Dr. Rajendra Prasad Government Medical College, Kangra, H. P. \\ ${ }_{5}^{5}$ Associate Professor, Department of Community Medicine, Dr. Rajendra Prasad Government Medical College, Kangra, H. P. \\ ${ }^{6}$ Professor and HOD, Dr. S. Radhakrishnan Medical College and Hospital, Hamirpur, Himachal Pradesh.
}

\begin{abstract}
BACKGROUND
ABSTRACT

Climate change is expected to alter the geographic and seasonal distributions of existing vectors and vector-borne diseases. Febrile illness is the most common presentation of these diseases and a public health problem affecting most of the people in developing world along with the prevailing burden of other communicable diseases.

Aims and Objectives- To study the seasonal trend analysis (2013 - 15) of fever cases admitted in a tertiary centre of Himachal Pradesh.
\end{abstract}

\section{MATERIALS AND METHODS}

A retrospective descriptive record-based analysis of fever cases admitted in medicine and paediatrics ward from 2013 - 15 of Dr. Rajendra Prasad Government Medical College, Kangra at Tanda, H.P.

\section{RESULTS}

Among febrile illnesses, fever of unknown origin is the leading cause of illness in all the three years. There is a resurgence of scrub typhus infection with increasing trend of cases in monsoon and post-monsoon season. Fever with neurological involvement was also recorded among young and productive age group in winter season with an upward trend.

\section{CONCLUSION}

There are only a limited number of studies from India reporting on the aetiology of fever and reliable surveillance data are not available; hence, a better insight in the epidemiology of febrile diseases will be helpful in order to target clinical work-up and treatment.

\section{KEYWORDS}

Trend, Febrile Illness, Himachal Pradesh.

HOW TO CITE THIS ARTICLE: Sharma P, Singh M, Gandhi MK, et al. Seasonal trend of fever cases seeking health care services at a tertiary care centre- an audit of retrospective data from sub-Himalayan region. J. Evolution Med. Dent. Sci. 2018;7(15):1862-1867, DOI: $10.14260 /$ jemds/2018/420

\section{BACKGROUND}

Infectious diseases are the leading causes of morbidity and death in tropical countries. The World Health Organisation (WHO) reports that each of the main infectious aetiologies (that is pneumonia, diarrhoea, HIV/ AIDS, malaria, tuberculosis (TB) and neonatal infections) cause between 1.05 and 0.24 million deaths respectively per year in low income countries. ${ }^{1}$

It occurs when various infectious and non-infectious processes interact with the host's defense mechanism. ${ }^{2}$ In most cases, fever is either due to identifiable microbiological agent or occurs during exposure to excessive environmental heat or during heavy physical work. ${ }^{3}$

'Financial or Other Competing Interest': None.

Submission 27-02-2018, Peer Review 23-03-2018,

Acceptance 30-03-2018, Published 09-04-2018.

Corresponding Author:

Dr. Mitasha Singh,

Assistant Professor,

Department of Community Medicine,

ESIC Medical College and Hospital,

Faridabad, NIT, NH-3, Haryana.

E-mail: mitasha.17@gmail.com

DOI: $10.14260 /$ jemds $/ 2018 / 420$
Febrile illness is the most common public health problem affecting most of the people in developing world, where sanitary conditions remain poor. Among febrile illnesses, malaria and dengue fever are the leading causes of illness in the Indian subcontinent. Recent reports from India and other neighbouring countries suggest that there is a resurgence of scrub typhus infection in this part of the world and that the resurgence is associated with considerable morbidity and mortality. ${ }^{4}$ Nevertheless, there are only a limited number of studies from India reporting on the aetiology of fever and reliable surveillance data are not available. $5 \mathrm{~A}$ better insight in the epidemiology of febrile diseases will be helpful in order to target clinical workup and treatment. In order to develop new applications which could potentially serve as treatments, we need to increase our knowledge on the way that hostpathogen interaction works. An aspect that has not been broadly experimentally addressed is the potential implications of fever on the interaction with the host with environment and the development of the infection. ${ }^{6}$ Therefore, this study will focus on fever as an important variable to be considered in infectious diseases research, focusing especially on seasonal variation of fever cases attendance at a tertiary care centre in rural area of Himachal Pradesh. 


\section{MATERIALS AND METHODS}

District Kangra is situated in southern escarpment of Himalayas. The altitude varies from 500 metres above mean sea level (AMSL) to around 5000 metres AMSL. It is encapsulated in the north by district Chamba and Lahaul Spiti in the south by Hamirpur and Una, and east by Mandi and in west by Gurdaspur district of Punjab. Kangra district is situated between $31^{\circ} 2$ to $32^{\circ} 5 \mathrm{~N}$ and $75^{\circ}$ to $77^{\circ} 45 \mathrm{E}$. The winter lasts from mid-December to mid-February, during which the temperature ranges from 0 to $20^{\circ} \mathrm{C}$. Summers last from April until June, and are hot (temp 25 to $38^{\circ} \mathrm{C}$ ) and dry. They are generally followed by a wet monsoon (July through September), which ends in autumn. A retrospective descriptive study of three consecutive years (2013 through 2015) was conducted at a tertiary care centre of Dr. Rajendra Prasad Government Medical College, Kangra at Tanda, Himachal Pradesh. Records of patients admitted in medicine and paediatrics ward with one of their chief complaints as fever were retrieved after permission from Institute's Ethical Department. Prior formal administrative approval was sought from the concerned authorities. An oral morning temperature of $>37.2^{\circ} \mathrm{C}\left(>98.9^{\circ} \mathrm{F}\right)$ or an evening temperature of $>37.7^{\circ} \mathrm{C}\left(>99.9^{\circ} \mathrm{F}\right)$ is defined as fever. Fever of unknown origin (FUO) was defined as (1) Temperatures of $>38.3^{\circ} \mathrm{C}(>$ $101^{\circ} \mathrm{F}$ ) on several occasions; (2) A duration of fever of $>3$ weeks and (3) Failure to reach a diagnosis despite 1 week of inpatient investigation. ${ }^{7}$ Data recorded included age, sex, residence, month of admission, any comorbid condition prevailing and the diagnosis or the system of the body involved. Data was entered in Microsoft Excel Sheet (2003 07) and trends of different illnesses was computed as proportion using Epi Info version 7. The trend analysis was done with Chi-Square test for trend at the five percent level of statistical significance.

\section{RESULTS}

The three-year record based data from tertiary care centre shows that out of all the cases with fever, majority were observed in 2015 (36\%) with an increasing trend from 201315. Monsoon season was observed with maximum attendance of fever cases in all the three years. The overall proportion of fever cases attending tertiary centre were observed with upward trend in winter, spring and monsoon season. A higher proportion of females admitted with fever had respiratory (11.7\%), liver $(15.5 \%)$ and neurological involvement $(14.3 \%)$ as compared to males $(17 \%, 13 \%$ and $12 \%$ respectively) (not in table). The proportion of females with fever attending tertiary care centre showed an upward trend both in monsoon (8.8\% increase) and autumn (5.9\% increase) in three years. In all the seasons, it was observed that majority of fever cases was reported in age group 16 - 30 years (Table 1a). Liver and spleen involvement (14.5\%) and neurological involvement (13.4\%) was observed and majority of cases with FUO contributing $32.9 \%$ of cases in 1630 years' age group in all three years (not in table). An upward trend was observed in this young age group in all seasons except in spring and summer season. There was significant increase in proportion of 1 - 15 years' age group admissions during monsoon (p: 0.01) and autumn (p: 0.02) (Table 1b). Children in this age group presented with scrub typhus in majority (78.9\%).

The study area (tertiary centre) was located in district Kangra, so majority of cases belonged to Kangra. In monsoon season, the fever cases from adjoining district Hamirpur showed a significant declining trend from 2013 (42.9\%) to 2015 (31.2\%) (p: 0.01). Also a significant upward trend of fever cases attending tertiary centre was observed from adjoining district Una in three years (55\% increase; p: 0.02). Una and Mandi contributed majority of scrub typhus cases; $11.6 \%$ and $8.4 \%$ respectively in all three years (not in table). The second major contributor of fever cases was district Chamba in winter, spring and summer season without any significant trend in three years. In monsoon and autumn, Hamirpur was the second major contributor of cases to the tertiary centre with a declining trend in three years (Table 1b).

The classification of fever cases has been done according to the organ system involved and the other major diagnosis of fever in the region and country. Majority of cases were labelled FUO in all the seasons followed by neurological involvement. An upward trend was observed among fever cases associated with neurological involvement in winter and monsoon seasons, but was statistically non-significant. A significant decline (p: 0.00) in neurological involvement with fever cases was observed in spring (March) and autumn (October and November) season in three years. In the three years in monsoon and autumn season, it has been observed that there had been a significant decline in fever cases with respiratory system, gastrointestinal system, liver and spleen involvement. There has been a significant increase in proportion of cases with dengue and scrub typhus in monsoon and autumn season (p: 0.00) (Table 1a and 1b). Death was reported in majority among elderly females (> 60 years) and no death was reported among males.

\begin{tabular}{|c|c|c|c|c|c|c|c|c|c|c|c|c|c|c|c|c|}
\hline \multicolumn{2}{|r|}{ Season } & \multicolumn{5}{|c|}{ Winter Season (December - February) } & \multicolumn{5}{|c|}{ Spring (March) } & \multicolumn{5}{|c|}{ Summer (April - June) } \\
\hline & & $\stackrel{m}{\stackrel{n}{N}}$ & 홍 & 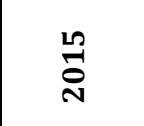 & $\stackrel{\text { 퓽 }}{\circ}$ & 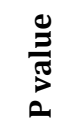 & $\stackrel{m}{\stackrel{\sim}{*}}$ & $\stackrel{+}{\text { ঠ }}$ & $\stackrel{\text { ㄴ }}{\stackrel{-}{\text { N }}}$ & $\stackrel{\pi}{\stackrel{\pi}{0}}$ & $\frac{0}{ٍ}$ & $\stackrel{m}{\stackrel{\sim}{\sim}}$ & 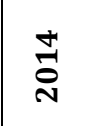 & $\stackrel{\text { Ln }}{\stackrel{2}{0}}$ & 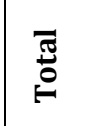 & 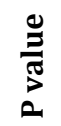 \\
\hline \multicolumn{2}{|r|}{ Total } & $\begin{array}{c}114 \\
(28.2)\end{array}$ & $\begin{array}{c}153 \\
(37.9)\end{array}$ & $137(33.9)$ & $\begin{array}{c}404 \\
(100)\end{array}$ & & $\begin{array}{c}65 \\
(31.3) \\
\end{array}$ & $\begin{array}{c}64 \\
(30.8) \\
\end{array}$ & $\begin{array}{c}79 \\
(38.0) \\
\end{array}$ & $\begin{array}{c}208 \\
(100) \\
\end{array}$ & & $\begin{array}{c}247 \\
(38.1)\end{array}$ & $\begin{array}{c}215 \\
(33.2) \\
\end{array}$ & $\begin{array}{c}186 \\
(28.7) \\
\end{array}$ & \begin{tabular}{|c|}
648 \\
$(100)$ \\
\end{tabular} & \\
\hline \multirow{2}{*}{ ڤ્ } & Male & $\begin{array}{c}50 \\
(26.0)\end{array}$ & $\begin{array}{c}68 \\
(35.4)\end{array}$ & $74(38.5)$ & $\begin{array}{c}192 \\
(100)\end{array}$ & 0.11 & $\begin{array}{c}32 \\
(31.4)\end{array}$ & $\begin{array}{c}27 \\
(26.5)\end{array}$ & $\begin{array}{c}43 \\
(42.2)\end{array}$ & $\begin{array}{c}102 \\
(100)\end{array}$ & 0.90 & $\begin{array}{c}114 \\
(36.9)\end{array}$ & $\begin{array}{c}100 \\
(32.4)\end{array}$ & $\begin{array}{c}95 \\
(30.7)\end{array}$ & $\begin{array}{c}309 \\
(100)\end{array}$ & 0.35 \\
\hline & Female & $\begin{array}{c}64 \\
(30.2) \\
\end{array}$ & $\begin{array}{c}85 \\
(40.1) \\
\end{array}$ & 63 (29.7) & $\begin{array}{c}212 \\
(100)\end{array}$ & & $\begin{array}{c}33 \\
(31.1) \\
\end{array}$ & \begin{tabular}{|c|}
37 \\
$(34.9)$ \\
\end{tabular} & $\begin{array}{c}46 \\
(34.0) \\
\end{array}$ & $\begin{array}{c}106 \\
(100) \\
\end{array}$ & & $\begin{array}{c}133 \\
(39.2)\end{array}$ & \begin{tabular}{|c|}
115 \\
$(33.9)$ \\
\end{tabular} & $\begin{array}{c}91 \\
(26.8) \\
\end{array}$ & \begin{tabular}{|c|}
339 \\
$(100)$ \\
\end{tabular} & \\
\hline \multirow{3}{*}{ 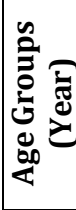 } & $1-15$ & 0 & 0 & $1(100)$ & $1(100)$ & 0.58 & 0 & 0 & $\begin{array}{c}1 \\
(100)\end{array}$ & $\begin{array}{c}1 \\
(100)\end{array}$ & 0.61 & 1 & 0 & 0 & $\begin{array}{c}1 \\
(100)\end{array}$ & 0.25 \\
\hline & $16-30$ & $\begin{array}{c}35 \\
(24.0)\end{array}$ & $\begin{array}{c}60 \\
(41.1)\end{array}$ & $51(34.9)$ & $\begin{array}{c}146 \\
(100)\end{array}$ & 0.26 & $\begin{array}{c}24 \\
(34.3) \\
\end{array}$ & $\begin{array}{c}24 \\
(34.3)\end{array}$ & $\begin{array}{c}22 \\
(31.4)\end{array}$ & $\begin{array}{c}70 \\
(100)\end{array}$ & 0.36 & $\begin{array}{c}86 \\
(35.7)\end{array}$ & $\begin{array}{c}81 \\
(33.6)\end{array}$ & $\begin{array}{c}74 \\
(30.7) \\
\end{array}$ & $\begin{array}{c}241 \\
(100)\end{array}$ & 0.50 \\
\hline & $31-45$ & $\begin{array}{c}35 \\
(28.2)\end{array}$ & $\begin{array}{c}48 \\
(38.7)\end{array}$ & 41 (33.1) & $\begin{array}{c}124 \\
(100)\end{array}$ & 0.86 & $\begin{array}{c}22 \\
(37.9) \\
\end{array}$ & $\begin{array}{c}17 \\
(29.3)\end{array}$ & $\begin{array}{c}19 \\
(32.8) \\
\end{array}$ & $\begin{array}{c}58 \\
(100)\end{array}$ & 0.30 & $\begin{array}{c}69 \\
(43.1)\end{array}$ & $\begin{array}{c}38 \\
(23.8)\end{array}$ & \begin{tabular}{|c}
53 \\
$(33.1)$ \\
\end{tabular} & $\begin{array}{c}160 \\
(100)\end{array}$ & 0.89 \\
\hline
\end{tabular}




\begin{tabular}{|c|c|c|c|c|c|c|c|c|c|c|c|c|c|c|c|c|}
\hline & $46-60$ & $\begin{array}{c}29 \\
(32.6)\end{array}$ & $\begin{array}{c}29 \\
(32.6)\end{array}$ & $31(34.8)$ & $\begin{array}{c}89 \\
(100)\end{array}$ & 0.75 & $\begin{array}{c}9 \\
(20.5)\end{array}$ & $\begin{array}{c}13 \\
(29.5)\end{array}$ & $\begin{array}{c}22 \\
(50.0)\end{array}$ & \begin{tabular}{|c|}
44 \\
$(100)$
\end{tabular} & 0.12 & $\begin{array}{c}47 \\
(33.8)\end{array}$ & \begin{tabular}{|c|}
59 \\
$(42.4)$
\end{tabular} & \begin{tabular}{|c|}
33 \\
$(23.7)$
\end{tabular} & \begin{tabular}{|c|}
139 \\
$(100)$
\end{tabular} & 0.88 \\
\hline & $>60$ & $\begin{array}{c}15 \\
(34.1)\end{array}$ & $\begin{array}{c}16 \\
(36.4)\end{array}$ & $13(29.5)$ & $\begin{array}{c}44 \\
(100)\end{array}$ & 0.36 & $\begin{array}{c}10 \\
(28.6)\end{array}$ & $\begin{array}{c}10 \\
(28.6)\end{array}$ & $\begin{array}{c}15 \\
(42.9)\end{array}$ & $\begin{array}{c}35 \\
(100)\end{array}$ & 0.70 & $\begin{array}{c}44 \\
(41.1)\end{array}$ & \begin{tabular}{|c|}
37 \\
$(34.6)$
\end{tabular} & \begin{tabular}{|c|}
26 \\
$(24.3)$
\end{tabular} & $\begin{array}{c}107 \\
(100)\end{array}$ & 0.35 \\
\hline \multirow{7}{*}{ 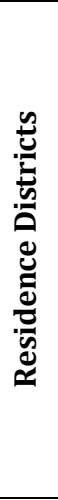 } & Kangra & $\begin{array}{c}81 \\
(28.8)\end{array}$ & $\begin{array}{c}106 \\
(37.7)\end{array}$ & $94(33.5)$ & $\begin{array}{c}281 \\
(100)\end{array}$ & 0.82 & $\begin{array}{c}43 \\
(30.7)\end{array}$ & $\begin{array}{c}40 \\
(28.6)\end{array}$ & $\begin{array}{c}57 \\
(40.7)\end{array}$ & $\begin{array}{c}140 \\
(100)\end{array}$ & 0.77 & $\begin{array}{c}175 \\
(39.9)\end{array}$ & \begin{tabular}{|c|}
153 \\
$(34.9)$
\end{tabular} & $\begin{array}{c}111 \\
(25.3)\end{array}$ & $\begin{array}{c}439 \\
(100)\end{array}$ & 0.28 \\
\hline & Hamirpur & $\begin{array}{c}11 \\
(36.7)\end{array}$ & $\begin{array}{c}11 \\
(36.7)\end{array}$ & $8(26.7)$ & $\begin{array}{c}30 \\
(100)\end{array}$ & 0.24 & $\begin{array}{c}5 \\
(22.7)\end{array}$ & $\begin{array}{c}7 \\
(31.8)\end{array}$ & $\begin{array}{c}10 \\
(45.5)\end{array}$ & $\begin{array}{c}22 \\
(100)\end{array}$ & 0.47 & $\begin{array}{c}18 \\
(28.1)\end{array}$ & $\begin{array}{c}25 \\
(39.1) \\
\end{array}$ & $\begin{array}{c}21 \\
(32.8)\end{array}$ & $\begin{array}{c}64 \\
(100)\end{array}$ & 0.21 \\
\hline & Chamba & $\begin{array}{c}13 \\
(20.0)\end{array}$ & $\begin{array}{c}26 \\
(40.0)\end{array}$ & $26(40.0)$ & $\begin{array}{c}65 \\
(100)\end{array}$ & 0.12 & $\begin{array}{c}15 \\
(40.5)\end{array}$ & $\begin{array}{c}13 \\
(35.1)\end{array}$ & $\begin{array}{c}9 \\
(24.3)\end{array}$ & $\begin{array}{c}37 \\
(100)\end{array}$ & 0.10 & $\begin{array}{c}39 \\
(37.9)\end{array}$ & $\begin{array}{c}26 \\
(25.2)\end{array}$ & $\begin{array}{c}38 \\
(36.9)\end{array}$ & $\begin{array}{c}103 \\
(100)\end{array}$ & 0.37 \\
\hline & Una & $2(25.0)$ & 0 & $6(75.0)$ & $8(100)$ & 0.18 & $\begin{array}{c}2 \\
(22.2)\end{array}$ & $\begin{array}{c}4 \\
(44.4) \\
\end{array}$ & $\begin{array}{c}3 \\
(33.3) \\
\end{array}$ & $\begin{array}{c}9 \\
(100)\end{array}$ & 0.96 & $\begin{array}{c}2 \\
(25.0) \\
\end{array}$ & $\begin{array}{c}3 \\
(37.5) \\
\end{array}$ & $\begin{array}{c}3 \\
(37.5) \\
\end{array}$ & $\begin{array}{c}8 \\
(100)\end{array}$ & 0.59 \\
\hline & Mandi & $6(33.3)$ & $9(50.0)$ & 3 (16.7) & $\begin{array}{c}18 \\
(100)\end{array}$ & 0.18 & 0 & 0 & 0 & 0 & & $\begin{array}{c}10 \\
(33.3) \\
\end{array}$ & \begin{tabular}{|c|}
8 \\
$(26.7)$ \\
\end{tabular} & \begin{tabular}{|c|}
12 \\
$(40.0)$ \\
\end{tabular} & $\begin{array}{c}30 \\
(100) \\
\end{array}$ & 0.35 \\
\hline & Bilaspur & $1(50.0)$ & $1(50.0)$ & 0 & $2(100)$ & 0.15 & 0 & 0 & 0 & 0 & & $\begin{array}{c}1 \\
(50.0) \\
\end{array}$ & 0 & \begin{tabular}{|c|}
1 \\
$(50.0)$ \\
\end{tabular} & $\begin{array}{c}2 \\
(100) \\
\end{array}$ & 0.79 \\
\hline & Pathankot & 0 & 0 & 0 & 0 & & 0 & 0 & 0 & 0 & & $2(100)$ & 0 & 0 & $\begin{array}{c}2 \\
(100)\end{array}$ & 0.04 \\
\hline \multirow{11}{*}{ 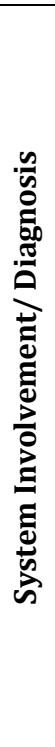 } & $\begin{array}{c}\text { Respiratory } \\
\text { System }\end{array}$ & $\begin{array}{c}19 \\
(38.8)\end{array}$ & $\begin{array}{c}17 \\
(34.7)\end{array}$ & $13(26.5)$ & $\begin{array}{c}49 \\
(100)\end{array}$ & 0.11 & $\begin{array}{c}6 \\
(17.1)\end{array}$ & $\begin{array}{c}12 \\
(34.3)\end{array}$ & $\begin{array}{c}17 \\
(48.6)\end{array}$ & $\begin{array}{c}35 \\
(100)\end{array}$ & 0.13 & $\begin{array}{c}34 \\
(45.3)\end{array}$ & $\begin{array}{c}26 \\
(34.7) \\
\end{array}$ & $\begin{array}{c}15 \\
(20.0)\end{array}$ & $\begin{array}{c}75 \\
(100)\end{array}$ & 0.09 \\
\hline & $\begin{array}{l}\text { Neurological } \\
\text { Involvement }\end{array}$ & $\begin{array}{c}20 \\
(29.0)\end{array}$ & $\begin{array}{c}25 \\
(36.2) \\
\end{array}$ & $24(34.8)$ & $\begin{array}{c}69 \\
(100)\end{array}$ & 0.94 & $\begin{array}{c}19 \\
(52.8)\end{array}$ & $\begin{array}{c}11 \\
(30.6)\end{array}$ & $\begin{array}{c}6 \\
(16.7) \\
\end{array}$ & $\begin{array}{c}36 \\
(100)\end{array}$ & 0.00 & $\begin{array}{c}40 \\
(34.5)\end{array}$ & $\begin{array}{c}48 \\
(41.4) \\
\end{array}$ & $\begin{array}{c}28 \\
(24.1) \\
\end{array}$ & $\begin{array}{c}116 \\
(100)\end{array}$ & 0.86 \\
\hline & \begin{tabular}{|c|} 
Gastrointestinal \\
System
\end{tabular} & $4(22.2)$ & $5(27.8)$ & $9(50.0)$ & $\begin{array}{c}18 \\
(100)\end{array}$ & 0.31 & $\begin{array}{c}2 \\
(15.4) \\
\end{array}$ & $\begin{array}{c}5 \\
(38.5) \\
\end{array}$ & $\begin{array}{c}6 \\
(46.2) \\
\end{array}$ & \begin{tabular}{|c|}
13 \\
$(100)$
\end{tabular} & 0.40 & $\begin{array}{c}11 \\
(39.3)\end{array}$ & \begin{tabular}{|c|}
10 \\
$(35.7)$ \\
\end{tabular} & $\begin{array}{c}7 \\
(25.0) \\
\end{array}$ & $\begin{array}{c}28 \\
(100)\end{array}$ & 0.67 \\
\hline & \begin{tabular}{|c|} 
Liver and Spleen \\
Involvement \\
\end{tabular} & $\begin{array}{c}12 \\
(26.7) \\
\end{array}$ & $\begin{array}{c}22 \\
(48.9) \\
\end{array}$ & $11(24.4)$ & $\begin{array}{c}45 \\
(100) \\
\end{array}$ & 0.46 & $\begin{array}{c}9 \\
(32.1) \\
\end{array}$ & $\begin{array}{c}8 \\
(28.6) \\
\end{array}$ & \begin{tabular}{|c|}
11 \\
$(39.3)$ \\
\end{tabular} & \begin{tabular}{|c|}
28 \\
$(100)$ \\
\end{tabular} & 0.92 & $\begin{array}{c}35 \\
(35.7) \\
\end{array}$ & \begin{tabular}{|c|}
30 \\
$(30.6)$ \\
\end{tabular} & \begin{tabular}{|c|}
33 \\
$(33.7)$ \\
\end{tabular} & $\begin{array}{c}98 \\
(100) \\
\end{array}$ & 0.44 \\
\hline & Renal System & $3(21.4)$ & $7(50.0)$ & $4(28.6)$ & $\begin{array}{c}14 \\
(100)\end{array}$ & 0.92 & $\begin{array}{c}2 \\
(25.0) \\
\end{array}$ & $\begin{array}{c}2 \\
(25.0) \\
\end{array}$ & \begin{tabular}{|c|}
4 \\
$(50.0)$ \\
\end{tabular} & \begin{tabular}{|c|}
8 \\
$(100)$ \\
\end{tabular} & 0.69 & $\begin{array}{c}6 \\
(40.0) \\
\end{array}$ & \begin{tabular}{|c|}
4 \\
$(26.7)$ \\
\end{tabular} & \begin{tabular}{|c|}
5 \\
$(33.3)$ \\
\end{tabular} & $\begin{array}{c}15 \\
(100) \\
\end{array}$ & 0.98 \\
\hline & Scrub Typhus & 0 & $2(9.1)$ & 20 (90.9) & $\begin{array}{c}22 \\
(100)\end{array}$ & 0.00 & 0 & 0 & \begin{tabular}{|c|}
4 \\
$(100)$ \\
\end{tabular} & \begin{tabular}{|c|}
4 \\
$(100)$
\end{tabular} & 0.06 & $\begin{array}{c}1 \\
(14.3) \\
\end{array}$ & 0 & \begin{tabular}{|c|}
6 \\
$(85.7)$ \\
\end{tabular} & $\begin{array}{c}7 \\
(100)\end{array}$ & 0.02 \\
\hline & Dengue Fever & 0 & 0 & $1(100)$ & $1(100)$ & 0.58 & 0 & 0 & 0 & 0 & & 0 & 0 & 1 & $\begin{array}{c}1 \\
(100)\end{array}$ & 0.47 \\
\hline & Malaria & 0 & 0 & 0 & 0 & & 0 & 0 & 0 & 0 & & 0 & 0 & 0 & 0 & \\
\hline & Tuberculosis & 0 & 0 & $1(100)$ & $1(100)$ & 0.58 & 1 & 0 & 0 & $\begin{array}{c}1 \\
(100)\end{array}$ & & 0 & 0 & 0 & 0 & \\
\hline & \begin{tabular}{|c|} 
Fever with other \\
Comorbidities \\
\end{tabular} & $\begin{array}{c}18 \\
(35.3) \\
\end{array}$ & $\begin{array}{c}21 \\
(41.2) \\
\end{array}$ & $12(23.5)$ & $\begin{array}{c}51 \\
(100) \\
\end{array}$ & 0.11 & $\begin{array}{c}8 \\
(34.8) \\
\end{array}$ & $\begin{array}{c}8 \\
(34.8) \\
\end{array}$ & \begin{tabular}{|c|}
7 \\
$(30.4)$ \\
\end{tabular} & \begin{tabular}{|c|}
23 \\
$(100)$ \\
\end{tabular} & 0.46 & $\begin{array}{c}38 \\
(40.8) \\
\end{array}$ & \begin{tabular}{|c|}
30 \\
$(32.3)$ \\
\end{tabular} & \begin{tabular}{|c|}
25 \\
$(26.9)$ \\
\end{tabular} & $\begin{array}{c}93 \\
(100) \\
\end{array}$ & 0.57 \\
\hline & \begin{tabular}{|c|} 
Fever with \\
Unknown Origin
\end{tabular} & $\begin{array}{c}37 \\
(28.0)\end{array}$ & $\begin{array}{c}54 \\
(40.9)\end{array}$ & $41(31.1)$ & $\begin{array}{c}132 \\
(100)\end{array}$ & 0.69 & $\begin{array}{c}18 \\
(30.0)\end{array}$ & $\begin{array}{c}18 \\
(30.0)\end{array}$ & \begin{tabular}{|c|}
24 \\
$(40.0)$
\end{tabular} & \begin{tabular}{|c|}
60 \\
$(100)$
\end{tabular} & 0.86 & $\begin{array}{c}82 \\
(38.1)\end{array}$ & \begin{tabular}{|c|}
67 \\
$(31.2)$ \\
\end{tabular} & \begin{tabular}{|c|}
66 \\
$(30.7)$
\end{tabular} & $\begin{array}{c}215 \\
(100)\end{array}$ & 0.69 \\
\hline
\end{tabular}

\begin{tabular}{|c|c|c|c|c|c|c|c|c|c|c|c|}
\hline \multicolumn{2}{|c|}{ Season } & \multicolumn{5}{|c|}{ Monsoon (July - September) } & \multicolumn{5}{|c|}{ Autumn (October - November) } \\
\hline & & 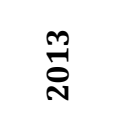 & $\underset{\sim}{\stackrel{\Xi}{D}}$ & 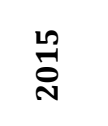 & 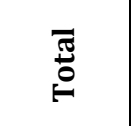 & 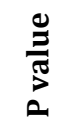 & $\stackrel{\text { N }}{\text { Dे }}$ & $\underset{\text { D }}{\stackrel{+}{D}}$ & 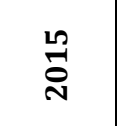 & 䒿 & $\begin{array}{l}0 \\
\frac{1}{3} \\
2 \\
2\end{array}$ \\
\hline \multicolumn{2}{|c|}{ Total } & $\begin{array}{c}447 \\
(34.0)\end{array}$ & $\begin{array}{c}342 \\
(26.0)\end{array}$ & $\begin{array}{c}525 \\
(40.0)\end{array}$ & $\begin{array}{l}1314 \\
(100)\end{array}$ & & $\begin{array}{c}263 \\
(36.1)\end{array}$ & $\begin{array}{c}203 \\
(27.8)\end{array}$ & $\begin{array}{c}263 \\
(36.1)\end{array}$ & $\begin{array}{c}729 \\
(100)\end{array}$ & \\
\hline \multirow{2}{*}{ હ } & Male & $\begin{array}{c}255 \\
(36.3)\end{array}$ & $\begin{array}{c}168 \\
(23.9)\end{array}$ & $\begin{array}{c}279 \\
(39.7)\end{array}$ & $\begin{array}{c}702 \\
(100)\end{array}$ & 0.24 & $\begin{array}{c}146 \\
(34.7)\end{array}$ & $\begin{array}{c}118 \\
(30.3)\end{array}$ & $\begin{array}{c}126 \\
(32.3)\end{array}$ & $\begin{array}{c}390 \\
(100)\end{array}$ & 0.07 \\
\hline & Female & $\begin{array}{c}192 \\
(31.4)\end{array}$ & $\begin{array}{c}174 \\
(28.4)\end{array}$ & $\begin{array}{c}246 \\
(40.2)\end{array}$ & $\begin{array}{c}612 \\
(100)\end{array}$ & & $\begin{array}{c}117 \\
(34.5)\end{array}$ & $\begin{array}{c}85 \\
(25.1)\end{array}$ & $\begin{array}{c}137 \\
(40.4)\end{array}$ & $\begin{array}{c}339 \\
(100)\end{array}$ & \\
\hline \multirow{5}{*}{ 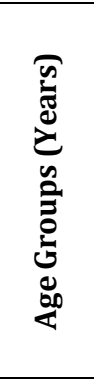 } & $1-15$ & 0 & $1(9.1)$ & $\begin{array}{c}10 \\
(90.9)\end{array}$ & $11(100)$ & 0.01 & 0 & 0 & $5(100)$ & $5(100)$ & 0.02 \\
\hline & $16-30$ & $\begin{array}{c}154 \\
(33.0)\end{array}$ & $\begin{array}{c}96 \\
(20.6)\end{array}$ & $\begin{array}{c}216 \\
(46.4)\end{array}$ & $\begin{array}{c}466 \\
(100)\end{array}$ & 0.12 & $\begin{array}{c}90 \\
(32.8)\end{array}$ & $\begin{array}{c}76 \\
(27.7)\end{array}$ & $\begin{array}{c}108 \\
(39.4)\end{array}$ & $\begin{array}{c}274 \\
(100)\end{array}$ & 0.30 \\
\hline & $31-45$ & $\begin{array}{c}130 \\
(37.9)\end{array}$ & $\begin{array}{c}88 \\
(25.7)\end{array}$ & $\begin{array}{c}125 \\
(36.4)\end{array}$ & $\begin{array}{c}343 \\
(100)\end{array}$ & 0.15 & $\begin{array}{c}75 \\
(37.9)\end{array}$ & $\begin{array}{c}43 \\
(21.7)\end{array}$ & $\begin{array}{c}80 \\
(40.4)\end{array}$ & $\begin{array}{c}198 \\
(100)\end{array}$ & 0.75 \\
\hline & $46-60$ & $\begin{array}{c}90 \\
(31.5)\end{array}$ & $\begin{array}{c}84 \\
(29.4)\end{array}$ & $\begin{array}{c}112 \\
(39.2)\end{array}$ & $\begin{array}{c}286 \\
(100)\end{array}$ & 0.78 & $\begin{array}{c}63 \\
(40.6) \\
\end{array}$ & $\begin{array}{c}52 \\
(33.5) \\
\end{array}$ & $\begin{array}{c}40 \\
(25.8) \\
\end{array}$ & $\begin{array}{c}155 \\
(100)\end{array}$ & 0.04 \\
\hline & $>60$ & $\begin{array}{c}73 \\
(35.1)\end{array}$ & $\begin{array}{c}73 \\
(35.1)\end{array}$ & $\begin{array}{c}62 \\
(29.8)\end{array}$ & $\begin{array}{c}208 \\
(100)\end{array}$ & 0.07 & $\begin{array}{c}35 \\
(36.1)\end{array}$ & $\begin{array}{c}32 \\
(33.0)\end{array}$ & $\begin{array}{c}30 \\
(30.9)\end{array}$ & $\begin{array}{c}97 \\
(100)\end{array}$ & 0.53 \\
\hline \multirow{3}{*}{ 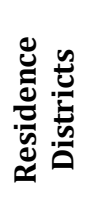 } & Kangra & $\begin{array}{c}309 \\
(33.3)\end{array}$ & $\begin{array}{c}240 \\
(25.9)\end{array}$ & $\begin{array}{c}378 \\
(40.8)\end{array}$ & $\begin{array}{c}927 \\
(100)\end{array}$ & 0.70 & $\begin{array}{c}172 \\
(35.0)\end{array}$ & $\begin{array}{c}134 \\
(27.3)\end{array}$ & $\begin{array}{c}185 \\
(37.7)\end{array}$ & $\begin{array}{c}491 \\
(100)\end{array}$ & 0.62 \\
\hline & Hamirpur & $\begin{array}{c}66 \\
(42.9) \\
\end{array}$ & $\begin{array}{c}40 \\
(26.0)\end{array}$ & $\begin{array}{c}48 \\
(31.2)\end{array}$ & $\begin{array}{c}154 \\
(100)\end{array}$ & 0.01 & $\begin{array}{c}43 \\
(44.8)\end{array}$ & $\begin{array}{c}26 \\
(27.1)\end{array}$ & $\begin{array}{c}27 \\
(28.1)\end{array}$ & $\begin{array}{c}96 \\
(100)\end{array}$ & 0.06 \\
\hline & Chamba & $\begin{array}{c}49 \\
(34.0)\end{array}$ & $\begin{array}{c}41 \\
(28.5)\end{array}$ & $\begin{array}{c}54 \\
(37.5)\end{array}$ & $\begin{array}{c}144 \\
(100)\end{array}$ & 0.70 & $\begin{array}{c}30 \\
(31.6)\end{array}$ & $\begin{array}{c}30 \\
(31.6)\end{array}$ & $\begin{array}{c}35 \\
(36.8)\end{array}$ & $\begin{array}{c}95 \\
(100)\end{array}$ & 0.61 \\
\hline
\end{tabular}




\begin{tabular}{|c|c|c|c|c|c|c|c|c|c|c|c|}
\hline & Una & $\begin{array}{c}1 \\
(5.0)\end{array}$ & $\begin{array}{c}7 \\
(35.0)\end{array}$ & $\begin{array}{c}12 \\
(60.0)\end{array}$ & $\begin{array}{c}20 \\
(100)\end{array}$ & 0.02 & $\begin{array}{c}1 \\
(14.3)\end{array}$ & $\begin{array}{c}4 \\
(57.1) \\
\end{array}$ & $2(28.6)$ & $\begin{array}{c}7 \\
(100)\end{array}$ & 0.83 \\
\hline & Mandi & $\begin{array}{c}20 \\
(32.3) \\
\end{array}$ & $\begin{array}{c}12 \\
(19.4) \\
\end{array}$ & $\begin{array}{c}30 \\
(48.4)\end{array}$ & $\begin{array}{c}62 \\
(100) \\
\end{array}$ & 0.40 & $\begin{array}{c}16 \\
(45.7) \\
\end{array}$ & $\begin{array}{c}6 \\
(17.1) \\
\end{array}$ & $\begin{array}{c}13 \\
(37.1) \\
\end{array}$ & $\begin{array}{c}35 \\
(100) \\
\end{array}$ & 0.50 \\
\hline & Bilaspur & $\begin{array}{c}2 \\
(33.3) \\
\end{array}$ & $\begin{array}{c}1 \\
(16.7)\end{array}$ & $\begin{array}{c}3 \\
(500)\end{array}$ & $\begin{array}{c}6 \\
(100)\end{array}$ & 0.95 & $1(33.3)$ & $\begin{array}{c}2 \\
(66.7) \\
\end{array}$ & 0 & $\begin{array}{c}3 \\
(100)\end{array}$ & 0.31 \\
\hline & Pathankot & 0 & $\begin{array}{c}1 \\
(100)\end{array}$ & 0 & $\begin{array}{c}1 \\
(100)\end{array}$ & 0.51 & 0 & $\begin{array}{c}1 \\
(50.0)\end{array}$ & $1(50.0)$ & $\begin{array}{c}2 \\
(100)\end{array}$ & 0.77 \\
\hline \multirow{11}{*}{ 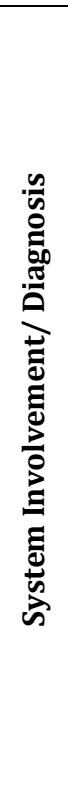 } & $\begin{array}{c}\text { Respiratory } \\
\text { System }\end{array}$ & $\begin{array}{c}44 \\
(44.0)\end{array}$ & $\begin{array}{c}31 \\
(31.0) \\
\end{array}$ & $\begin{array}{c}25 \\
(25.0) \\
\end{array}$ & $\begin{array}{c}100 \\
(100)\end{array}$ & 0.00 & $\begin{array}{c}21 \\
(42.0) \\
\end{array}$ & $\begin{array}{c}20 \\
(40.0) \\
\end{array}$ & $9(18.0)$ & $\begin{array}{c}50 \\
(100)\end{array}$ & 0.04 \\
\hline & $\begin{array}{l}\text { Neurological } \\
\text { Involvement }\end{array}$ & $\begin{array}{c}41 \\
(28.9) \\
\end{array}$ & $\begin{array}{c}43 \\
(30.3)\end{array}$ & $\begin{array}{c}58 \\
(40.8) \\
\end{array}$ & $\begin{array}{c}142 \\
(100)\end{array}$ & 0.46 & $\begin{array}{c}39 \\
(53.4) \\
\end{array}$ & $\begin{array}{c}18 \\
(24.7) \\
\end{array}$ & $\begin{array}{c}16 \\
(21.9)\end{array}$ & $\begin{array}{c}73 \\
(100) \\
\end{array}$ & 0.00 \\
\hline & $\begin{array}{c}\text { Gastrointestinal } \\
\text { System }\end{array}$ & $\begin{array}{c}27 \\
(45.8) \\
\end{array}$ & $\begin{array}{c}18 \\
(30.5)\end{array}$ & $\begin{array}{c}14 \\
(23.7) \\
\end{array}$ & $\begin{array}{c}59 \\
(100)\end{array}$ & 0.01 & $\begin{array}{c}5 \\
(21.7) \\
\end{array}$ & $\begin{array}{c}9 \\
(39.1) \\
\end{array}$ & $9(39.1)$ & $\begin{array}{c}23 \\
(100)\end{array}$ & 0.40 \\
\hline & $\begin{array}{c}\text { Liver and Spleen } \\
\text { Involvement }\end{array}$ & $\begin{array}{c}88 \\
(45.1)\end{array}$ & $\begin{array}{c}45 \\
(23.1)\end{array}$ & $\begin{array}{c}62 \\
(31.8) \\
\end{array}$ & $\begin{array}{c}195 \\
(100)\end{array}$ & 0.00 & $\begin{array}{c}56 \\
(53.8) \\
\end{array}$ & $\begin{array}{c}32 \\
(30.8) \\
\end{array}$ & $\begin{array}{c}16 \\
(15.4) \\
\end{array}$ & $\begin{array}{c}104 \\
(100) \\
\end{array}$ & 0.00 \\
\hline & Renal System & $\begin{array}{c}6 \\
(30.0) \\
\end{array}$ & $\begin{array}{c}7 \\
(35.0) \\
\end{array}$ & $\begin{array}{c}7 \\
(35.0) \\
\end{array}$ & $\begin{array}{c}20 \\
(100)\end{array}$ & 0.86 & $\begin{array}{c}4 \\
(66.7) \\
\end{array}$ & 0 & $2(33.3)$ & $\begin{array}{c}6 \\
(100) \\
\end{array}$ & 0.23 \\
\hline & Scrub Typhus & $\begin{array}{c}8 \\
(6.6) \\
\end{array}$ & $\begin{array}{l}12 \\
(9.9)\end{array}$ & $\begin{array}{c}101 \\
(83.5) \\
\end{array}$ & $\begin{array}{c}121 \\
(100)\end{array}$ & 0.00 & $\begin{array}{c}7 \\
(6.8) \\
\end{array}$ & $\begin{array}{l}18 \\
(17.5) \\
\end{array}$ & $\begin{array}{c}78 \\
(75.7) \\
\end{array}$ & $\begin{array}{c}103 \\
(100) \\
\end{array}$ & 0.00 \\
\hline & Dengue Fever & $\begin{array}{c}12 \\
(19.7)\end{array}$ & $\begin{array}{c}2 \\
(3.3)\end{array}$ & $\begin{array}{c}47 \\
(77.0)\end{array}$ & $\begin{array}{c}61 \\
(100)\end{array}$ & 0.00 & $\begin{array}{c}21 \\
(27.6)\end{array}$ & $\begin{array}{c}8 \\
(10.5) \\
\end{array}$ & $\begin{array}{c}47 \\
(61.8)\end{array}$ & $\begin{array}{c}76 \\
(100)\end{array}$ & 0.00 \\
\hline & Malaria & $\begin{array}{c}3 \\
(60.0)\end{array}$ & 0 & $\begin{array}{c}2 \\
(40.0)\end{array}$ & $\begin{array}{c}5 \\
(100)\end{array}$ & 0.35 & $\begin{array}{c}1 \\
(33.3)\end{array}$ & $\begin{array}{c}2 \\
(66.7)\end{array}$ & 0 & $3(100)$ & 0.31 \\
\hline & Tuberculosis & 0 & $1(100)$ & 0 & $1(100)$ & 0.51 & 0 & 0 & $1(100)$ & $1(100)$ & 0.56 \\
\hline & $\begin{array}{c}\text { Fever with other } \\
\text { Comorbidities }\end{array}$ & $\begin{array}{c}77 \\
(38.7) \\
\end{array}$ & $\begin{array}{c}59 \\
(29.6)\end{array}$ & $\begin{array}{c}63 \\
(31.7) \\
\end{array}$ & $\begin{array}{c}199 \\
(100)\end{array}$ & 0.04 & $\begin{array}{c}44 \\
(39.6) \\
\end{array}$ & $\begin{array}{c}42 \\
(37.8) \\
\end{array}$ & \begin{tabular}{|c|}
25 \\
$(22.5)$ \\
\end{tabular} & $\begin{array}{c}111 \\
(100)\end{array}$ & 0.04 \\
\hline & \begin{tabular}{c|} 
Fever with \\
Unknown Origin
\end{tabular} & $\begin{array}{c}141 \\
(34.4)\end{array}$ & $\begin{array}{c}124 \\
(30.2)\end{array}$ & $\begin{array}{c}145 \\
(35.4)\end{array}$ & $\begin{array}{c}410 \\
(100)\end{array}$ & 0.29 & $\begin{array}{c}65 \\
(36.5) \\
\end{array}$ & $\begin{array}{c}54 \\
(30.3) \\
\end{array}$ & $\begin{array}{c}59 \\
(33.1)\end{array}$ & $\begin{array}{c}178 \\
(100)\end{array}$ & 0.60 \\
\hline & & & & & & & & & & & \\
\hline
\end{tabular}

\section{DISCUSSION}

Himachal Pradesh is a mountainous state in northern India, situated in the outer Himalayas with altitudes $350-7,000 \mathrm{~m}$ above sea level. It is the least urbanised state in India. During the rainy season, areas at lower altitudes have an average temperature of $20^{\circ} \mathrm{C}$ to $35^{\circ} \mathrm{C}$, which is conducive to the spread of arthropod vectors. In monsoon and post monsoon season, there is overgrowth of scrub grass required for laying eggs of mite and transmission of scrub typhus. Similar observations have been reported in present study and other studies.8,9 This seasonal pattern should alert the treating physicians to consider scrub typhus in the list of differentials that commonly includes only dengue and malaria during these months. ${ }^{10}$ An outbreak investigation of FUO in 2003 was investigated in Shimla, HP (Indira Gandhi Medical College) and scrub typhus was confirmed through lab diagnosis in most of the districts of the state.11 An analysis from a tertiary centre in Rajasthan showed that both dengue and malaria follow the same seasonal pattern in both years with increased incidence of scrub typhus (24\% in 2012 to $35.23 \%$ in 2013) and dengue (47\% in 2012 to $56 \%$ in 2013). ${ }^{12}$

\section{Arthropod-Borne Diseases}

Dengue, another arthropod-borne viral infection, its vector (Aedes) was once found to be scanty at altitude 500 metres to 1000 metres and above. But in recent years, cases of dengue is being reported from sub-Himalayan region with three cases reported in 2010 followed by zero in 2011, 73 in 2012 and 89 in 2013.13 In the current analysis also, an increasing proportion of cases of dengue has been observed from 201315 from July through November. The incidence of dengue cases at high altitudes indicates that such areas would not remain free of hosts in the light of the modifying effects to the ecosystem associated with human influences on both arboviruses and their vectors local and global scale. Also the cases could be from neighbouring low lying plain areas (districts Una and Pathankot) referred to the government run tertiary care centre.

For most Anopheles vector species of malaria, the optimal temperature range for their development lies within $20^{\circ} \mathrm{C}$ to $30^{\circ} \mathrm{C}$. However, transmission of $\mathrm{P}$. vivax requires a minimum average temperature of $15^{\circ} \mathrm{C}$ and transmission by $\mathrm{P}$. falciparum, requires a minimum temperature of $19^{\circ} \mathrm{C} .1^{14}$ The Global Circulation Model and climate change scenario suggests that number of people in developing countries that are likely to be at risk of malaria infection will increase by $5 \%-15 \%$, because of climate change. The areas that are expected to have the most increase in malaria transmission are ones that is at the fringes of transmission now. The population in these areas having low levels of immunity is likely to experience recurrence of epidemics unless appropriate control efforts are put in place to effectively reduce the impact of the epidemics. Evidence shows that areas which are now free of malaria such as Himachal Pradesh, where the average altitude is above $1800 \mathrm{~m}$ may become malaria prone under the changed climate conditions in the 2050s.15 In the current analysis the fever cases diagnosed with malaria constitute a small proportion of cases, but in areas with nil reporting even one case is alarming. 


\section{Water-Borne Diseases}

High-altitude areas $(>12,500 \mathrm{~m})$ do not harbour specific infectious agents. ${ }^{16}$ Enteric infections are the leading cause of illness in travelers regardless of altitude. Persistence of enteric infections in three years in the current study in all seasons is evident with predominance in monsoon season. A study in the Himalayas found that $14 \%$ of a cohort of foreign outdoor trekkers developed gastroenteritis. ${ }^{17}$ Also this part of the state has a large number of pilgrims visiting during monsoons contributing to the enteric infections and hepatitis

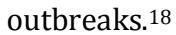

\section{Airborne Infection}

Bacterial meningitis, endemic in many developing country settings such as the Indian subcontinent, is the commonest cause of fever with neurological involvement. An increasing trend has been observed in winter and monsoon season in cases of fever with neurological involvement in this study. A time series analysis of bacterial meningitis shows that three main groups of countries could be distinguished. First, in countries with latitudes above $30^{\circ} \mathrm{N}$ (Europe, North America and China), the mean timing of Neisseria meningitidis activity (the pathogen most represented in the time-series) was located in winter in January to February. Second, in all countries of the African meningitis belt (Between $-5^{\circ} \mathrm{N}$ and $20^{\circ} \mathrm{N}$ ), the seasonal timing of bacterial meningitis was situated in February to March during the dry season. Third, in countries below $-20^{\circ} \mathrm{N}$ (South America, Oceania and South Africa), the incidence of disease caused by any of the pathogens peaked June to July (winter season). ${ }^{19}$ So our study area falls in the first belt where winter cases are explained by N. meningitides activity. A causal role of environmental factors in meningitis is still unknown. It has been postulated that climatic conditions could facilitate pathogen invasion by damaging the nasopharyngeal mucosa. 20

\section{Age and Gender Variation}

Female attendance for seeking health care with fever as chief complaint observed an upward trend in three years, particularly in monsoon and autumn season. This could be due to majority of women working outdoors in fields could have probably been subjected to vectors or bacterial/ viral pathogens during the vulnerable seasons. Younger and productive age group (16 - 30 years) also accounted for majority of admissions with fever. This is probably due to increased outdoor activities among this age group, which leads to frequent contraction of arthropod-borne and foodborne infections.

\section{Seasonal Variation}

FUO is still the diagnosis with which major admissions occur and monsoon season accounts for majority of admissions in all the three years. The finding is supported by similar study from other part of the country. ${ }^{21}$ This is different from the observation made at Government Medical College, Chandigarh, which revealed that the overall reporting of communicable diseases was significantly more during winter and summer compared to the monsoon season. ${ }^{22}$ Temperature, humidity, ultraviolet radiation (UVR), flora and fauna all change with seasons, and consequently cause a change in the frequency of occurrence of various diseases.

\section{Role of Health Care Delivery System}

The current analysis was conducted among the admitted cases of tertiary centre, which may lead to underestimation of various endemic communicable diseases of the region (eg. Tuberculosis), probably due to institutional framework of rural health care delivery system. The first point of care being primary health centre where majority of cases are encountered and treated on outpatient basis and if needed referred to first referral point, that is community health centres. $^{23}$ Tertiary centre ideally should cater to the cases needing specialist and advanced care. The patient's right for choice of facility for health services is the reason behind high load of patients at tertiary centre, who can be managed at primary or secondary level. In spite of the best efforts on the part of the physician, diagnosing a case of fever will sometimes be a challenge. In our country, prescription rules are often not enforced and patients are free to try any 'quick fix' strategies. ${ }^{24}$ The burden of various emerging and reemerging diseases could be reduced if we take measures to detect the changes in their trend through the implementation of surveillance programs in this part of the world, as has been carried out in other countries.

\section{CONCLUSION}

The surveillance system in every health care centre should be strengthened to study the seasonal variation of the diseases over a longer period of time spanning over years to draw a precise statement regarding the consistency of the seasonal variation. Further this will be helpful to the health planners for the judicious allocation of the limited resources and further exploration of the association of the factors with the occurrence of the diseases for the achievement of the optimal health of the people.

\section{REFERENCES}

[1] World Health Organisation: the 10 leading causes of death by broad income group (2008). WHO, Geneva, 2011.

[2] Kliegman RM, Behrman RE. Fever. In: Behrman RE, Kliegman RM, Nelson WE, eds. Nelson textbook of paediatrics. 14th edn. Philadelphia: Saunders 1992: p. 647-51.

[3] Al-Eissa YA, Al- Sanie AM, Al-Alola SA, et al. Parental perceptions of fever in children. Ann Saudi Med 2000;20(3-4):202-5.

[4] Issac R, Varghese GM, Mathai E, et al. Scrub typhus: prevalence and diagnostic issues in rural southern India. Clin Infect Dis 2004;39(9):1395-6.

[5] John TJ, Dandona L, Sharma VP, et al. Continuing challenge of infectious diseases in India. Lancet 2011;377(9761):252-69.

[6] Blauenfeldt $T$, Wagner $D$, Aabye $M$, et al. Thermostability of IFN- $\gamma$ and IP-10 release assays for latent infection with Mycobacterium tuberculosis: a TBnet study. Tuberculosis 2016;98:7-12.

[7] Longo DL, Fauci AS, Kasper DL, et al. Fever and hyperthermia. Harrison's Principles of internal medicine. 18 ${ }^{\text {th }}$ edn. New York: McGraw-Hill 2012.

[8] Kamarasu K, Malathi M, Rajagopal V, et al. Serological evidence for wide distribution of spotted fevers \& typhus fever in Tamil Nadu. The Indian Journal of Medical Research 2007;126(2):128-30. 
[9] Vivekanandan M, Mani A, Priya YS, et al. Outbreak of scrub typhus in Pondicherry. The Journal of the Association of Physicians of India 2010;58:24-8.

[10] Gupta N, Chaudhry R, Kabra SK, et al. In search of scrub typhus: a prospective analysis of clinical and epidemiological profile of patients from a tertiary care hospital in New Delhi. Advances in Infectious Diseases 2015;5:140-7.

[11] Sharma A, Mahajan S, Gupta ML, et al. Investigation of an outbreak of scrub typhus in the Himalayan region of India. Jpn J Infect Dis 2005;58(4):208-10.

[12] Sharma R, Ahmed S, Gupta S. Comparative evaluation of seasonal fevers in last 2 years at a tertiary care hospital in North India. Int J Curr Microbiol App Sci 2014;3(7):631-4.

[13] Government of India. Annual Report. National Programmes under NRHM. Chapter 6. p88. 2014. www.mohfw.nic.in.

[14] Sharma VP, Bos R. Determinants of Malaria in SouthAsia. In: Casman EA, Dowlatabadi $\mathrm{H}$, eds. The contextual determinants of Malaria. Washington, DC: Resources for the Future 2003.

[15] Bhattacharya S, Sharma C, Dhiman RC, et al. Climate change and Malaria in India. Current Science 2006;90(3):369-75.

[16] Zafren K, Honigman B. High-altitude medicine. Emerg Med Clin North Am 1997;15(1):191-222.

[17] Basnyat B, Lemaster J, Litch JA. Everest or bust: a cross sectional, epidemiological study of acute mountain sickness at 4243 meters in the Himalayas. Aviat Space Environ Med 1999;70(9):867-73.
[18] Katoch V. Outbreak investigation of acute viral hepatitis in Kangra valley, Himachal Pradesh, India, 2014-2015. International Journal of Infectious Disease 2016;45(Suppl 1):230.

[19] Paireau J, Chen A, Broutin H, et al. Seasonal dynamics of bacterial meningitis: a time-series analysis. Lancet Glob Health 2016;4(6):370-7.

[20] Greenwood B. Manson Lecture. Meningococcal meningitis in Africa. Trans $\mathrm{R}$ Soc Trop Med Hyg 1999;93(4):341-53.

[21] Kumari R, Nath B, Midha T, et al. Morbidity profile and seasonal variation of diseases in a primary health center in Kanpur district: a tool for the health planners. J Family Med Prim Care 2012;1(2):86-91.

[22] Sharma MK, Bhatnagar T, Goel NK, et al. Operationalisation of surveillance of communicable diseases in Chandigarh. J Commun Dis 2005; 37(3):197-202.

[23] World Health Organization. Country Health System Profile. Geneva, WHO. http://www.searo.who.int/EN/Section313/Section15 19_10853.htm. Last accessed on December 31, 2016.

[24] Ittyachen AM, Ramachandran R. Study of acute febrile illness: a 10-year descriptive study and a proposed algorithm from a tertiary care referral hospital in rural Kerala in southern India. Tropical Doctor 2015;45(2):114-7. 